\title{
Erratum: Polarized Fermi gases at finite temperature in the BCS-BEC crossover [Phys. Rev. A 90, 053629 (2014)]
}

\author{
Pierre-Alexandre Pantel, Dany Davesne, and Michael Urban \\ (Received 24 June 2016; published 12 July 2016)
}

DOI: 10.1103/PhysRevA.94.019901

The phase-transition lines between the normal and the FFLO phase, shown as the blue dashed lines in Figs. 6 and 7 of our article, are not correct.

Actually, it is not possible to calculate these lines within the present theoretical framework. This can be seen as follows. Consider first the transition from the normal to the BCS ( $q=$ $0)$ superfluid phase. In this case, the $T$ matrix $\Gamma(\Omega, q)$ develops for $T \rightarrow T_{c}\left(\right.$ or $\left.\delta \mu^{*} \rightarrow \delta \mu_{c}^{*}\right)$ a pole at $\Omega=0, q=0$. In spite of this pole, the correction to the density $\rho^{(1)}$ and the self-energy $\Sigma_{\sigma}$ remain well defined in the limit $T \rightarrow T_{c}$ (or $\delta \mu^{*} \rightarrow \delta \mu_{c}^{*}$ ), because a pole at $q=0$ has zero weight in the integral over $\mathbf{q}$ in the calculation of $\rho^{(1)}$ and in the integral over $\mathbf{k}^{\prime}=\mathbf{q}-\mathbf{k}$ in the calculation of $\Sigma_{\sigma}$. This is, however, no longer true for the phase transition towards the FFLO phase where the pole appears at a finite value $q \neq 0$. Then, $\rho^{(1)}$ and $\Sigma_{\sigma}$ diverge in the limit $T \rightarrow T_{c}$ (or $\left.\delta \mu^{*} \rightarrow \delta \mu_{c}^{*}\right)$.

The phase-transition lines in Figs. 6 and 7 of our article were computed by extrapolating $\rho^{(1)}$ from two values of $\left(\delta \mu^{*}, T\right)$ close to the phase transition line but still in the normal phase to the point $\left(\delta \mu_{c}^{*}, T_{c}\right)$ on the phase-transition line. But as discussed above, this extrapolation cannot give a meaningful result in the case $q \neq 0$.

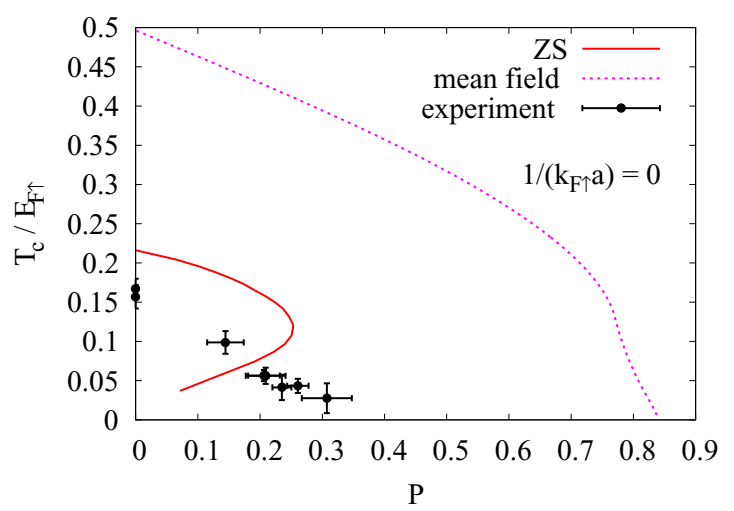

FIG. 6. Critical temperature $T_{c}$ in units of $E_{F \uparrow}=k_{F \uparrow}^{2} /(2 m)$ vs. polarization $P$ for a unitary Fermi gas $\left(1 /\left(k_{F \uparrow} a\right)=0\right)$. The transition towards a BCS- (or Sarma-) like superfluid, computed with improved numerical precision, is shown as the solid line, while the transition line towards a FFLO-like phase shown in Fig. 6 of our article has been removed. The $T_{c}$ vs. $P$ curve obtained without the correction $\rho^{(1)}$ (BCS mean-field result) is shown as the dotted line. The experimental data are from Y.-I. Shin, C. H. Schunck, A. Schirotzek, and W. Ketterle, Nature 451, 689 (2008), except the two points at $P=0$, which are from S. Nascimbène, N. Navon, K. J. Jiang, F. Chevy, and C. Salomon, Nature 463, 1057 (2010) and M. J. H. Ku, A. T. Sommer, L. W. Cheuk, and M. W. Zwierlein, Science 335, 563 (2012).
Furthermore, when approaching the tricritical point, i.e., the point on the phase-transition line where one passes from the normal-BCS transition to the normal-FFLO transition, $\rho^{(1)}$ gets larger and larger. This seems plausible since, as discussed above, $\rho^{(1)}$ diverges beyond the tricritical point. As a consequence, both the ratio $T / E_{F \uparrow}$ with $E_{F \uparrow} \propto\left(\rho_{\uparrow}^{(0)}+\rho^{(1)}\right)^{2 / 3}$ and the polarization $P=\left(\rho_{\uparrow}^{(0)}-\rho_{\downarrow}^{(0)}\right) /\left(\rho_{\uparrow}^{(0)}+\rho_{\downarrow}^{(0)}+2 \rho^{(1)}\right)$ are strongly reduced. From a numerical point of view, the extrapolation gets more and more difficult as one has to go very close to $T_{c}$ (or $\delta \mu_{c}^{*}$ ) to get the correct extrapolated result.

We include the revised version of Fig. 6 here, where we display the phase diagram for the unitary limit, obtained with improved numerical precision. In this case, if $\rho^{(1)}$ really diverges at the tricritical point, this point should lie at $T / E_{F \uparrow}=$ 0 and $P=0$. However, in spite of the improved numerics, we were not able to push our calculation beyond the endpoint of the phase-transition line shown in Fig. 6.

In the revised version of Fig. 7 given here, we show the phase diagram for the case $1 /\left(k_{F \uparrow} a\right)=-0.5$. Here, $\rho^{(1)}$ cannot become arbitrarily high, since $1 /\left(k_{F \uparrow} a\right)$ is fixed and $k_{F \uparrow} \propto$ $\left(\rho_{\uparrow}^{(0)}+\rho^{(1)}\right)^{1 / 3}$. Therefore the endpoint of the phase transition line (i.e., where it reaches the tricritical point) lies at finite values of the polarization $P$ and the ratio $T / E_{F \uparrow}$. This figure has not changed substantially compared to Fig. 7 of our article except that the line corresponding to the FFLO transition has been removed.

We thank P. Pieri for pointing out to us the possible problem in the calculation of $\rho^{(1)}$ at the transition to the FFLO phase.

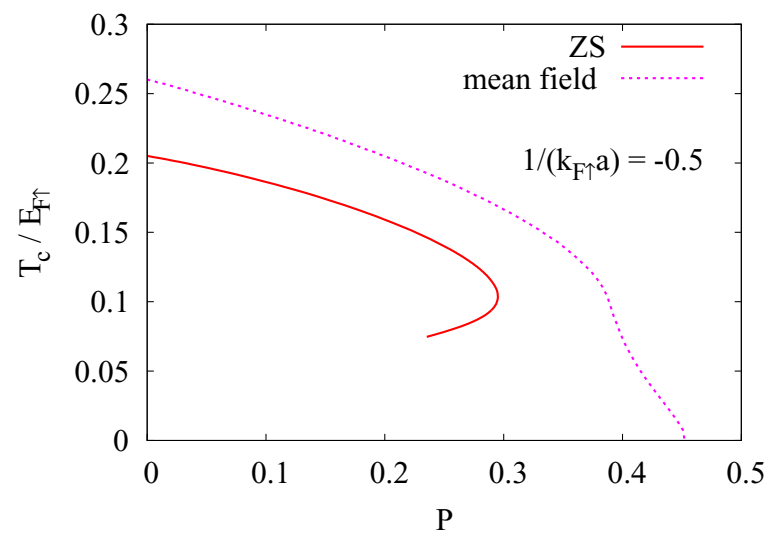

FIG. 7. Same as Fig.6, but for $1 /\left(k_{F \uparrow} a\right)=-0.5$. 